\title{
Modeling iSE emission for Ion Beam Imaging
}

\author{
David C Joy ${ }^{1}$ and Brendan Griffin ${ }^{2}$. \\ 1. Dept. of Materials Science and Engineering, University of Tennessee, Knoxville, TN. USA \\ 2. Center for Microscopy, University of Western Australia, Crawley WA, Australia 6009
}

Electron microscopy is an important and widely used scientific tool, but some aspects of its application fall far short of what normally would be regarded as acceptable practice. For example, little attention has been given to the need to obtain, validate, and make available, accurate physical data. For example even though secondary electrons (SE) have been intensively studied and employed for over a century it is still impossible for a microscopist to find a reference which can give the magnitude of the SE yield from any element or material, at any energy, with an uncertainty of less than about $50 \%$.

Regrettably the problem for ion induced SE (iSE) interactions is even worse because in addition to the usual number of target materials there are now also a multitude of different ions of interest which must be considered. Because it is not practical to be able to expect to rapidly all the desired experimental data that is needed then some other approach is required. It will be shown that, given iSE yield data $\delta_{\text {iSE }}$ as a function of the energy $\mathrm{E}$ of a given incident ion beam, the iSE yield from that same material but using any other choice of ion can then be reliably predicted using a Monte Carlo method. The IONiSE program [1] is a Monte Carlo simulation of ion transport and ion induced secondary electron data for generation in solids. The iSE yield is computed using the Bethe-Salow 'continuous slowing-down' model which incorporates two parameters, $\varepsilon$, which is the rate of energy transfer from the ion, and $\lambda$ which describes the escape probability of the secondary electron. For any ion, and for any target material, these two parameters can be deduced by fitting to experimental iSE yield vs beam energy profiles. We have now obtained $\varepsilon, \lambda$ value pairs from a total of forty elements, for one, or in many case more than one, of the ions of interest $(\mathrm{H}+, \mathrm{He}+, \mathrm{Ne}+, \mathrm{Ar}+$ and $\mathrm{Ga}+)$ and within the energy range from $10 \mathrm{keV}$ to $10 \mathrm{MeV}$. This data shows conclusively that these $\varepsilon, \lambda$ parameters depend only on the target material and not on the choice of ion. Consequently it is now possible to insert iSE yield data values that are missing in published data, and even to predict iSE yield curves for elemental and ion beam combinations that have not yet been experimental determined.

The method described above is limited in application to pure elements, so the urgently required next step is to be able to extend the IONiSE Monte Carlo program so that it can also model and predict the iSE yield from compounds and alloys as a function of energy. Initial attempts to measure and fit iSE yield data from even simple binary compounds were unsuccessful, most probably because the experimental data was of poor quality, varying by a factor of two to three times from one sample of a material to another. The reasons for this are not clear but certainly the state of cleanliness of the surface is one factor of high importance. A $30 \mathrm{keV} \mathrm{He}+$ ion beam impinging, for example, on a metal surface will lose a significantly high fraction of its energy as it travels through a few nanometers of surface oxides or residual finger grease, reducing both the energy deposited in the target as the ion enters and the intensity of the generated iSE signal as it travels back towards the surface to escape. For example, published iSE yield profiles [2] from a $\mathrm{He}+$ beam incident on to pure iron, and on to stainless steel, shows a very significant variation in the magnitude and form of the iSE yield curves as a function of the beam energy in the two cases despite their similar chemistries, differences attributed by these authors to the presence of a nano-surface oxide layer. 
One response to this behavior has been to accept it as being correct and then to employ a multiparameter polynomial expression to fit the available data. This "solution" has meaning only within the energy range that has been experimentally investigated, and no validity at all outside those limits.

We have now successfully obtained fits to experimental iSE yield data for a few compound materials, by placing special attention on the cleanliness of the sample surface. The procedure has been to treat the compound as an "elemental" solid with an effective mean atomic number $Z_{\text {Eff }}$ calculated from weighted average of the atomic numbers of elemental constituents of the material. For example, as shown in figure 2, an excellent fit to the data for stainless steel is obtained in this way. As before, it is not guaranteed that this procedure has any validity outside the energy range within which the original data was obtained and fitted, and it remains true that even a very thin surface film - such as one less than $5 \mathrm{~nm}$ thick - might have a significant effect on the magnitude of the parameters because the ion induced iSE yield is at a maximum at the entrance surface - while for electrons the maximum SE yield occurs close to the end of the beam range. A systematic attempt is being made to measure iSE yields from a wide range of compound materials to better answer the questions raised by this effect.

\section{References:}

[1 ] R Ramachandra et al., Ultramicroscopy, 109, (2009), p748

[2] L. Dietz and J Sheffield, J. of Applied Physics, 46, (1979), p4361

[3] This work was partially supported by SRC Contract 2011-OJ-2122, Manager Dr. R.Havemann

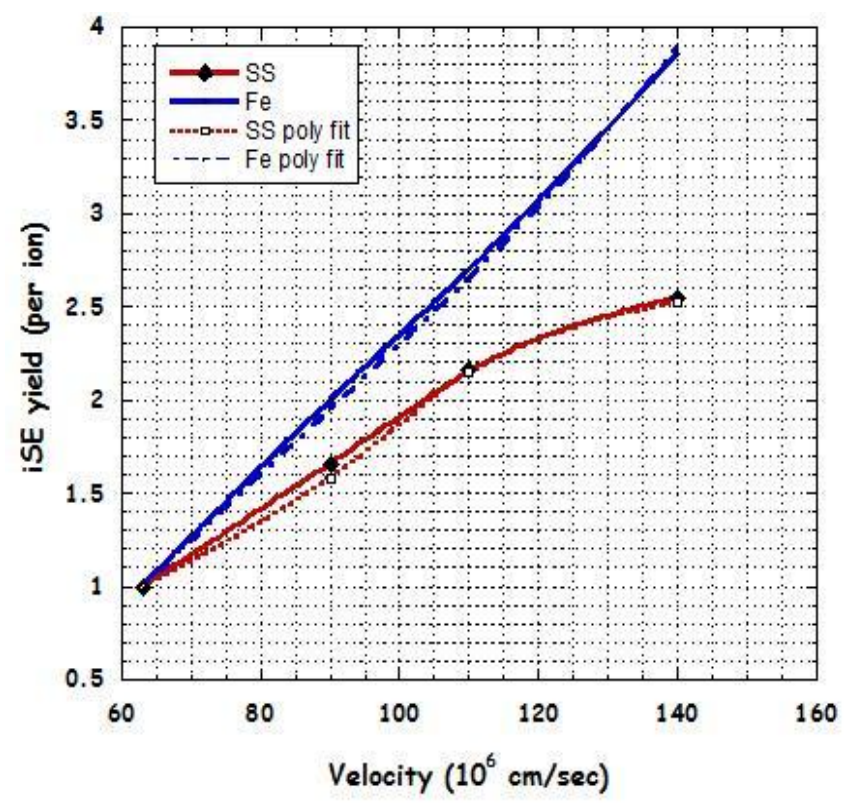

Figure 1. Plot of the $\mathrm{He}+$ generated iSE yield from pure iron and from stainless steel as a function of the incident ion velocity. Data from ref.\#2

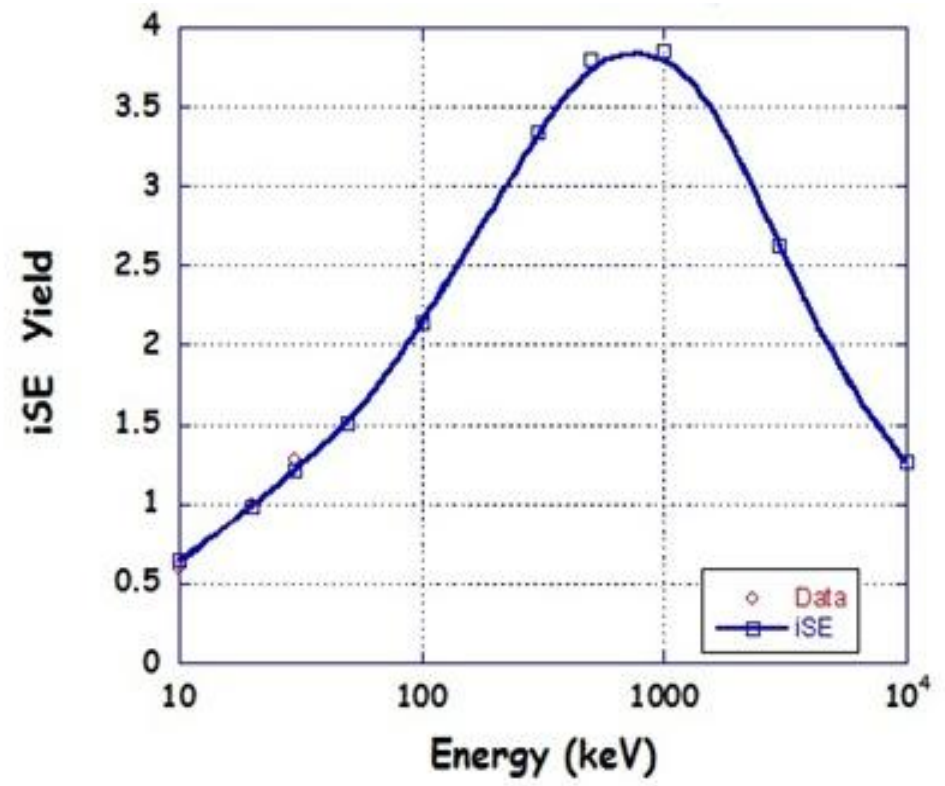

Figure 2. iSE yield vs $\mathrm{He}+$ beam energy for stainless steel as fitted by IONiSE using hybrid parameters 